\title{
SUBJECTIVE AND OBJECTIVE ASSESSMENT OF NEUROMUSCULAR FATIGUE IN FEMALE PAINTERS
}

\author{
K. Jansen ${ }^{1}$, M. Luik², V. Viljasoo ${ }^{2}$, J. Ereline ${ }^{1}$, \\ H. Gapeyeva ${ }^{1}$, M. Pääsuke ${ }^{1}$ \\ ${ }^{1}$ Institute of Exercise Biology and Physiotherapy, \\ University of Tartu, Tartu, Estonia \\ ${ }^{2}$ Institute of Technology, Estonian University of Life Sciences, \\ Tartu, Estonia
}

\section{ABSTRACT}

The aim of this study was to evaluate subjectively and objectively neuromuscular fatigue in female painters before and after a working day. The subjects $(n=11)$ were female painters aged $22-60$ years. The subjects completed a questionnaire and gave a subjective evaluation on muscle fatigue sensation in hands, trunk, back and lower limbs according to Borg's Category Ratio (CR-10) scale. Thereafter they performed 3-minute test of painting a wall, in the course of which the electromyographical (EMG) power spectral median frequency (MF) slope for biceps brachii, trapezius, deltoid and infraspinatus muscles was measured. The results indicated a significant change in the subjective muscle fatigue sensation in hands by $40 \%$, lower limbs by $54 \%$ and trunk by $57 \%$ after the working day, compared to the beginning of the working day. EMG power spectral MF slope of the measured muscles did not differ significantly during the 3-minute wall coloring test before and after working day. It was concluded that subjective muscle fatigue sensation in hands, lower limbs and trunk was higher after the working day whereas it was less pronounced in the back. Muscle fatigue evaluated objectively by MF slope of EMG power spectrum from biceps brachii, trepezius, deltoid and infraspinatus muscles was not evident during the wall coloring test before and after the working day.

Key words: subjective, objective, muscle fatigue, electromyography 


\section{INTRODUCTION}

The work-induced complaints of upper limbs can be described as disorders that involve neck, shoulders, arms, elbows, wrists, hands and fingers. They are caused by repetitive movements of fingers, hands or arms, including pushing, pulling, drawing, reaching, turning, raising, gripping or hitting. Most of all, this concerns professions such as painter, decorator, riveter, pneumatic tools operator and user of desktop computer [4]. Salem et al. [7] measured the conformity of profession and the musculoskeletal diseases amongst 147 construction workers by a questionnaire. It was found that four factors connected with work (the working environment, mental work, performance and contentment) were closely related with the musculoskeletal symptoms, musculature and stress.

Garg et al. [3] studied shoulder girdle muscle strength of one hand in women when working with hands elevated above the head. It was found that women, especially in those professions that require working with hands elevated above the head, have little strength in their shoulder muscles. Chow and Dickerson [2] studied shoulder strength of females while sitting and standing as a function of hand location and force direction. It was found that there is a significant decrease in shoulder strength of females compared to males. Direction has the greatest affect on shoulder strength when working at or aboveshoulder level. The optimal position that maximizes shoulder strength is vertically downwards.

The aim of this study was to evaluate subjectively and objectively neuromuscular fatigue in female painters before and after a working day. Measurements were carried out at the place of work at the beginning of the working day and after it. 


\section{MATERIAL AND METHODS}

\section{Subjects}

Eleven females working as painters with (mean \pm SD) age of $43.4 \pm 12.3$ years participated in this study. The height, body mass and body mass index of the subjects were $163.5 \pm 9.1 \mathrm{~cm}, 78.3 \pm 16.2 \mathrm{~kg}$ and $29.3 \pm 5.4 \mathrm{~kg} / \mathrm{m}^{2}$, respectively, and their length of employment as painter was $19.5 \pm 13.9$ years. The subjects were randomly selected and participated in the research voluntarily. The questionnaires and the measurements were completed in May and June, 2008. Larger facilities were chosen as the site of the measurements. Nine subjects were surveyed at the 1st stage of construction at the Tartu University Maarjamõisa centre of medical facilities and two subjects at the Tasku fashion and entertainment centre in downtown Tartu. The subjects were familiarized with the essence and the aims of the survey.

\section{Data collection}

The measurements were conducted at the beginning and at the end of the working day at the site where the workers were employed. So the subjects did not have to leave the site and the working rhythm was disturbed as little as possible, thus yielding more reliable results. The height and body mass of the subjects were measured at the site with metal anthropometer and electronic scales, respectively. The body mass index $\left(\mathrm{kg} / \mathrm{m}^{2}\right)$ of the subjects was also calculated. The questions in the questionnaire were in Estonian and Russian. In the course of the research, subjects completed the questionnaire first. Subjective muscle fatigue sensation in hands, trunk, back and lower limbs was estimated with a psychophysical rating scale (Borg's CR-10 Scale). The scale included numbers from 0 to 10 . Perceived exertion was estimated in the following way: 0-2 weak, 3-4 moderate, 5-7 strong, 8-10 extremely strong fatigue. Thereafter, a 3-minute test of painting the wall (Figure 1) was conducted. 


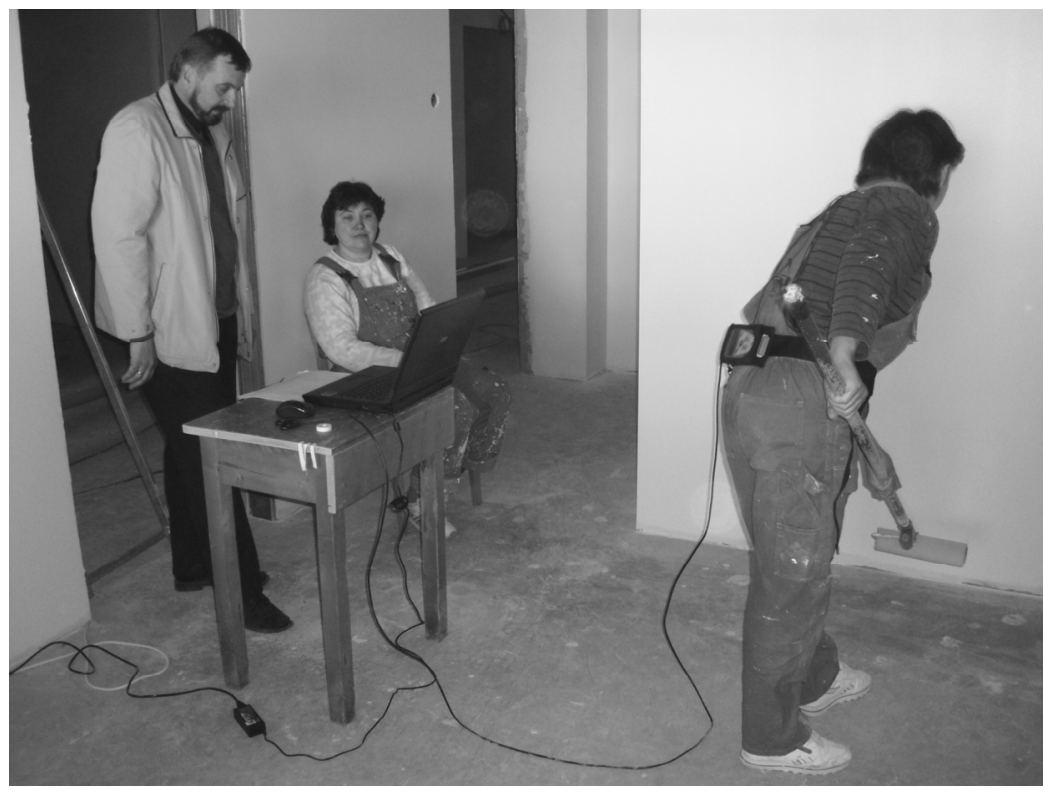

Figure 1. Performing the dosed wall coloring test.

Muscle fatigue was objectively estimated by electromyographic (EMG) activity registered from biceps brachii, trapezius, deltoid and infraspinatus muscles using 8-channel electromyograph ME 6000 (Mega Electronics, Finland). The data gained were processed with the computer application MegaWin (2007). EMG power spectrum median frequency (MF) slope $(\% / \mathrm{kg} \cdot \mathrm{min})$ was calculated by the formula:

$$
M F_{\text {slope }}=\frac{\left(M F_{b}-M F_{a}\right) \cdot t}{M F_{b} \cdot P \cdot 60} \cdot 100 \text {, }
$$

whereas $\mathrm{MF}_{\mathrm{b}}$ is a EMG power spectrum median frequency at the beginning of the working day, $\mathrm{MF}_{\mathrm{a}}$ is a median frequency after the working day, $\mathrm{t}$ is a test time (3-minutes) and $\mathrm{P}$ is the weight of the extension pole $(3.2 \mathrm{~kg})$. 


\section{Statistical analysis}

When processing the data, the usual methods were applied for calculating the mean and the standard deviation $( \pm \mathrm{SD})$. The significance of the changes for the group of subjects at the beginning of the working day and after it has been analyzed by using the pairwise t-test. The differences of the means of the group, as well as the significance of the changes for the group of subjects at the beginning of the working day and after, were evaluated on the basis of the Student t-test, $p<0.05$ was chosen as the statistically significant level.

\section{RESULTS}

The results indicated a significant subjective muscle fatigue sensation evaluated according to the Borg $(\mathrm{CR}-10)$ scale in hands $(\mathrm{p}<0.05)$, lower limbs $(\mathrm{p}<0.01)$ and trunk $(\mathrm{p}<0.05)$ before and after working day (Figure 2). At the end of the working day, the estimation of subjective muscle fatigue sensation increased by $40 \%$ for hands, by $30 \%$ for back, by $57 \%$ for trunk and by $54 \%$ for lower limbs as compared to the beginning of the working day.

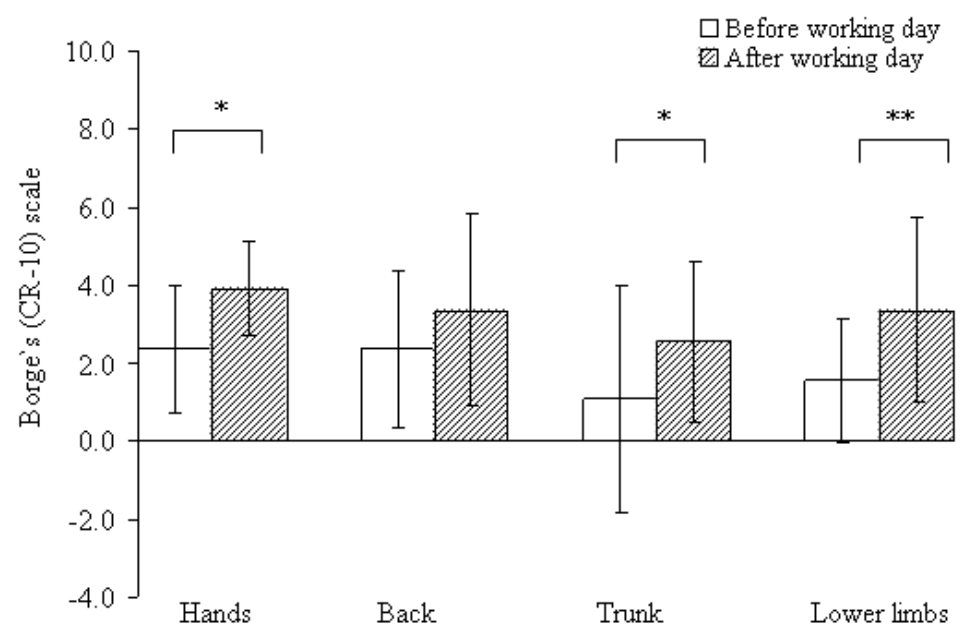

Figure 2. Subjective muscle fatigue sensation, estimated with a Borg's Category Ratio (CR-10) scale at the beginning of the working day and after it (mean $\pm \mathrm{SD}$ ); ${ }^{*} \mathrm{p}<0.05 ; * * \mathrm{p}<0.01$. 
EMG power spectrum median frequency (MF) slope (Figure 3) of the measured muscle groups during wall coloring test did not differ significantly $(\mathrm{p}>0.05)$ at the beginning and after the working day.

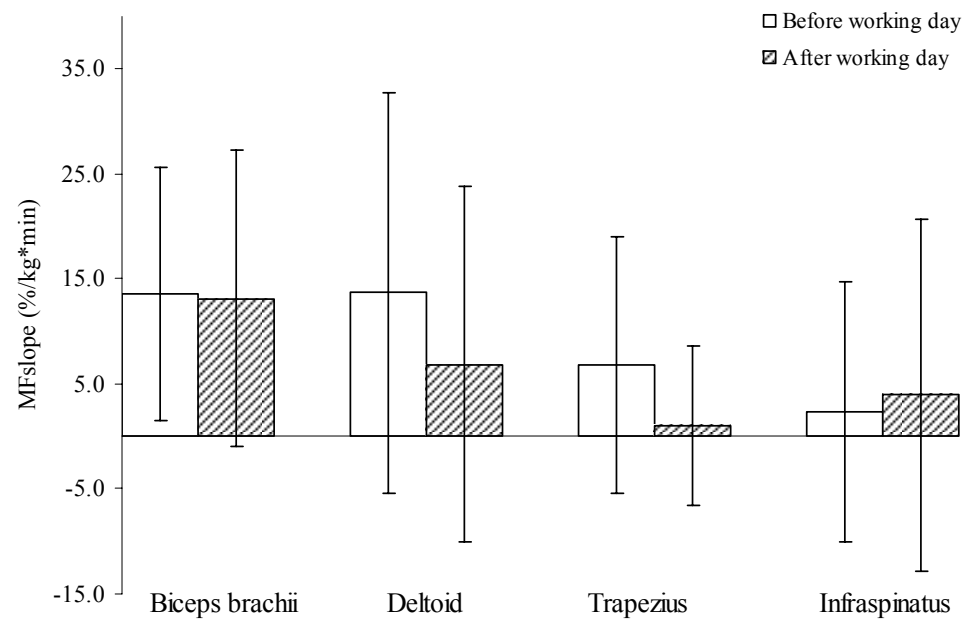

Muscles

Figure 3. EMG power spectrum median frequency (MF) slope at the beginning of the working day and after it (mean $\pm \mathrm{SD}$ ).

\section{DISCUSSION}

On the basis of the Borg's Category Ratio (CR-10) scale [1], the female workers experienced subjectively muscle fatigue sensation after the working day more in hands, lower limbs, trunk and less in the back. A change in the subjective muscle fatigue sensation of hands, lower limbs and trunk before and after work was evident and this result deserves attention. It is known that physical stress and musculoskeletal discomfort when working can be alleviated and prevented by selecting the right tool that reduces the physical stress in the worker's fingers and hands to minimum, and he or she needs to use less energy for working. A correctly selected tool also reduces jolting, repulse, and vibration [6].

During the 3-minute wall coloring test, EMG power spectral activity MF slope did not change significantly but there was objec- 
tively estimated muscle fatigue when comparing the beginning of the working day and the end of the working day. This can be related to the fact that at the beginning of the working day, the muscles have not yet reached their working capacity. The MF slope of the EMG power spectrum of the observed group revealed that in case biceps brachii, deltoid and trapezius muscles, this indicator was moderately lower $(p>0.05)$ at the end of the working day. But in case of infraspinatus muscles MF slope it was moderately higher $(p>0.05)$ at the beginning of the working day than it was at the end of the working day. This fact indicates that female painters tend to be more overloaded in the hands and shoulder region and less in the back.

The subjective muscle fatigue sensation of lower limbs may be due to the fact that painters have to stand throughout the working day mostly on concrete floor, which lacks the amortization that would reduce the jolting. Comfortable working shoes of good quality are of great help for reducing such overload in case of workers (including painters), who have to work standing all day long. A pair of shoes that does not suit one's foot can cause problems enduring for years [5].

The study revealed that painters were using different working styles, whereas the working tool was the same for everyone and noone customized it for herself. It is also important to emphasize that $82 \%$ of the workers had not been instructed in terms of ergonomics.

Proceeding from the data gained in the course of the research, that the painters had mostly the shoulder girdle and hands overload, it is recommended that they perform stretching exercises before and during the working day. A worker should customize the tools proceeding from his/her own anthropometrical measures and use suitable means of protection. This requires the corresponding instructive materials and instructing the workers by a trained specialist. In case of physical work, it is advisable to make short breaks (5..10 minutes) every hour to avoid the problems caused by overload. Similarly important are the consumption of the necessary volume of liquids and following the balanced diet, since the current research and the previous ones have proven that in women, the neck and shoulder region are considerably burdened in case of physical work, indicating substantial expense of energy.

In conclusion, this study indicated that subjective muscle fatigue sensation in female painters was higher after the working day in hands, lower limbs and trunk, less pronounced in the back. Muscle fatigue, evaluated objectively by EMG power spectrum MF slope 
from biceps brachii, trapezius, deltoid and infraspinatus muscles was not evident during 3-minute wall coloring test before and after the working day.

\section{REFERENCES}

1. Borg G. (1990) Psychophysical scaling with applications in physical work and the perception of exertion. Scand. J. Work Environ. Health. 16: $55-58$

2. Chow A. Y., Dickerson C. R. (2008) Shoulder strength of females while sitting and standing as a function of hand location and force direction. Appl. Ergon. 40: 303-308

3. Garg A., Hegmann K. T., Kapellusch J. (2005) Maximum one-handed shoulder strength for overhead work as a function of shoulder posture in females. Occup. Ergon. 5: 131-140

4. Hughes P., Ferrett E. (2003) Introduction to Health and Safety at Work. The Handbook for the NEBOSH National General Certificate, Oxford

5. Kane M. W. (1987) Understanding Health. The second edition, New Jersey

6. Marras W. S., Karwowski W. (2005) The Occupational Ergonomics Handbook. Second edition. In: R. G. Randwin (ed), (Design and evaluation of handtools. Madison: Univ. of Wisconsin. 1

7. Salem O., Sobeih T. M., Genaidy A., Shell R., Bhattacharya A., Succop P. (2008) Work compatibility and musculoskeletal disorders in the construction industry. Hum. Factors Ergon. \& Manufact. 18: $230-252$

\section{Correspondence to:}

Kristjan Jansen

Institute of Exercise Biology and Physiotherapy

University of Tartu

Jakobi 5

Tartu 51014

Estonia

E-mail: jansen@ut.ee 